\title{
Les affaissements miniers dans le bassin ferrifère lorrain : quand le territoire re-politise la gestion du risque
}

François Duchêne

\section{(2) OpenEdition \\ Journals}

Édition électronique

URL : http://journals.openedition.org/developpementdurable/7073

DOI : 10.4000/developpementdurable.7073

ISSN : $1772-9971$

Éditeur

Association DD\&T

\section{Référence électronique}

François Duchêne, «Les affaissements miniers dans le bassin ferrifère lorrain : quand le territoire repolitise la gestion du risque », Développement durable et territoires [En ligne], Dossier 11 | 2008, mis en ligne le 30 octobre 2008, consulté le 19 avril 2019. URL : http://journals.openedition.org/ developpementdurable/7073 ; DOI : 10.4000/developpementdurable.7073

Ce document a été généré automatiquement le 19 avril 2019

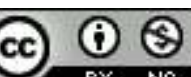

Développement Durable et Territoires est mis à disposition selon les termes de la licence Creative Commons Attribution - Pas d'Utilisation Commerciale 4.0 International. 


\title{
Les affaissements miniers dans le bassin ferrifère lorrain : quand le territoire re-politise la gestion du risque
}

\author{
François Duchêne
}

1 Les affaissements miniers constituent un risque particulier, entre nature - ils mettent en jeu la mécanique des sols - et industrie - les désordres des sous-sols sont causés par une extraction minière, régie par une organisation de type industriel. Compte tenu des modes d'exploitation, les risques d'affaissement ont toujours existé depuis les débuts de la mine. Ils vont devenir une "affaire d'Etat » avec la fin de la période d'extraction et donc la fin de la prise en charge des dégâts par les compagnies minières. Plus précisément, il s'agit d'une transformation du statut de ce risque, contenu jusque là dans la catégorie « risques liés au travail » et qui va basculer vers celle des « risques environnementaux ».

2 Ce qui nous importe ici plus particulièrement est que ce risque a été amplifié et travaillé par le contexte économique et social dans lequel il a changé de statut, lors de la désindustrialisation de la Lorraine et la fin de l'exploitation ferrifère. Autrement dit, à travers cette étude de cas, nous souhaitons montrer que les risques territoriaux n'existent pas de façon autonome, sectorielle et ex-nihilo, mais qu'ils sont généralement ancrés dans un contexte historique local qu'il convient de décrypter si l'on souhaite les traiter et les prévenir efficacement dans toutes leurs dimensions. Nous l'écrivions, «le clivage trop souvent réifié entre "initiés" et "profanes", la focalisation sur l'événement catastrophe et la faible prise en compte de la dimension territoriale constituent des obstacles à la compréhension du risque et de la manière dont il est constitué par la formation sociale concernée " (Duchêne, Morel Journel et alii, $2004: 7$ ). Ces propos, tenus dans un contexte de risques d'inondation, valent aussi pour la situation présentée ici. En effet, nous nous situons dans une approche constructiviste des risques, et nous nous appuyons sur la définition qu'en propose Thierry Coanus, à savoir que «le risque [serait] la représentation d'un danger, non encore matérialisé - bien qu'ayant pu survenir auparavant. Et gérer un risque, dans cette optique, 
c'est: repérer un danger, et donc entre autres choses le définir, en fonction des savoirs, voire des croyances disponibles; anticiper son occurrence, c'est-à-dire opérer dans le temps une projection mentale; prévenir son occurrence, en effectuant un certain nombre d'actions, individuelles et/ou collectives» (2006: 21). Dans le cas qui nous intéresse, le sous-sol ferrifère lorrain a travaillé, physiquement comme socialement, d'une part dans un cadre réglementaire mouvant et d'autre part selon des cadres de production différents en fonction des exploitants. Aujourd'hui, l'ennoyage des bassins, depuis le milieu des années 1990 à l'issue de l'exploitation, modifie l'équilibre des sous-sols. Les affaissements qui se produisent actuellement résultent des effets de ces différents facteurs. Mais nous allons voir qu'on ne peut isoler les phénomènes physiques d'affaissement et d'effondrement du sol des contextes historique, politique, économique et social particuliers qu'ont constitué l'industrialisation puis la désindustrialisation massive de ce territoire frontalier.

3 Notre propos s'appuiera sur une enquête effectuée pour l'essentiel dans un cadre pédagogique ${ }^{1}$, et réalisée auprès de différents acteurs lorrains ${ }^{2}$, concernés d'une manière ou d'une autre par la question des affaissements. Nous reviendrons dans un premier temps sur le contexte historique d'exploitation des mines de fer lorrain. Nous décrirons ensuite la façon dont la question des affaissements, dès lors qu'elle est devenue une affaire publique, s'est chargée de toute l'histoire sociale et politique dont la mine était porteuse. Nous verrons enfin le mode de traitement «techniciste » du problème par les agents de l'Etat qui en avaient la charge, comme une manière de mettre à distance le social, et donc la dimension proprement territoriale ${ }^{3}$ de ces risques, dont ils ne savaient visiblement que faire.

1. Un contexte d'exploitation particulièrement mouvant

4 Les affaissements miniers lorrains résultent d'une exploitation qui n'a rien ni d'uniforme ni d'homogène. Elle s'est étendue sur plus d'un siècle et, dans cette période, a été menée dans des cadres économiques et réglementaires particulièrement mouvants.

1.1 Une industrialisation tardive

5 S'il y a eu de très longue date une exploitation ferrifère et des forges dans la région, la Lorraine industrielle, telle qu'elle a été érigée en figure quasi-mythique par les maîtres de forge dans le courant du $\mathrm{XX}^{\mathrm{e}}$ siècle, ne s'est constituée en réalité qu'assez tardivement. Tout au long du XIXe siècle, il existait une petite industrie métallurgique dispersée sur le territoire. Elle était en particulier le fait d'une ancienne noblesse terrienne fonctionnant à côté de l'agriculture en employant pour l'essentiel des paysans-ouvriers. D'ailleurs, l'exploitation des minières (à ciel ouvert) constituait une activité de terrassement peu différenciée de celle du travail agricole, et la mine attirait encore beaucoup de paysans. Il existait aussi, au début du XVIII ${ }^{\mathrm{e}}$ siècle, quelques familles traditionnelles de maitres de forge, parmi lesquelles les Wendel, qui allaient devenir de véritables dynasties (Rondeau, Baudin, 1979). De plus récents sidérurgistes étaient issus de familles bourgeoises ayant acquis des biens fonciers après la Révolution. Mais une concurrence assez vive opposait encore ces maîtres de forge, bloqués dans l'exploitation industrielle des minières, à une vieille noblesse terrienne désireuse de conserver sa main-d'œuvre. Et « pendant cinquante ans, l'aristocratie parvint, en mobilisant l'ensemble des forces rurales, à empêcher un réel décollage industriel dans la région » (Noiriel, $1984: 28$ ).

Plusieurs éléments concoururent à l'émergence du fait industriel lorrain à la fin du XIX siècle. L'ouverture de la voie ferrée Paris - Charleville - Longwy (vers 1860), d'abord, permit de substituer le coke au bois de forêt, et fit chuter le prix de revient de la fonte lorraine. Ensuite, diverses techniques parmi lesquelles celle de Bessemer, en 1853, 
permettant de transformer la fonte en acier, puis surtout le procédé de déphosphoration Thomas, inventé en 1877, permirent une exploitation de grande ampleur de la minette, minerai oolithique extrait du sous-sol, plus pauvre en fer ${ }^{4}$ que celui de surface exploité jusqu'alors. La bourgeoisie sidérurgique montante se saisit rapidement de cette opportunité pour acquérir des concessions en nombre. En effet, depuis les décrets napoléoniens industrialistes de 1810, le sous-sol est considéré comme propriété de l'Etat. Les services étatiques délivraient donc des concessions pour ouvrir une mine, là où les minières, régies par des privilèges terriens datant d'avant la Révolution, ne dépendaient généralement pas d'eux. Et le corps montant des Ingénieurs des mines, défenseur de l'industrialisation du pays, arbitra autant que possible en faveur des nouveaux maîtres de forge, dans les conflits les opposant encore ici ou là à l'aristocratie agraire. Ainsi purent se constituer rapidement des puissances capitalistiques locales, en capacité d'investir dans de gros équipements industriels, par un jeu d'alliances successives et complexes.

Puis, vers 1900, après une intense activité de prospection, un nouveau bassin ferrifère fut découvert dans le canton de Briey, frontalier de l'Allemagne depuis 1871. D'une superficie de 22.000 ha, il fut considéré à l'époque comme la seconde réserve ferrifère au monde derrière le bassin du Lac Supérieur aux Etats-Unis : «La Meurthe-et-Moselle surclassa désormais tous les autres départements puisque de $38 \%$ en 1880 , sa part dans la production nationale de fer passa à $91 \%$ en $1910 »$ (Noiriel, $1984: 66$ ).

Le système productif industriel était désormais en place: en amont, on trouvait l'extraction minière, généralement en sous-sol mais dans une couche peu profonde ; puis les complexes sidérurgiques comprenaient un haut fourneau au coke (transformant le minerai en fonte), un convertisseur (de fonte en acier) et un laminoir (permettant de dénaturer l'acier en « demi-produits » ou en produits marchands). Ce système technique d'exploitation n'a pas connu de bouleversement jusqu'à la Deuxième Guerre mondiale. Par la suite, «ce n'est pas tellement sur les procédés de base qu'ont porté les transformations technologiques, mais surtout sur la mécanisation des manutentions et sur la connaissance des processus physico-chimiques de transformation de la matière » (Noiriel, $1984: 43$ ).

1.2. Des systèmes socio-spatiaux spécifiques

9 Pendant près de cinquante années, de 1871 à 1919, la Meurthe-et-Moselle a été un département frontalier à la suite de l'annexion de l'Alsace-Lorraine par l'Allemagne. Sans doute cette annexion, l'avant-dernière puisqu'elle fut renouvelée de 1940 à 1944, constitua-t-elle côté français un véritable sentiment de frontière. Fernand Braudel (1990: 334) rappelle en effet, jusque-là, la longue porosité de ces limites de la France : « Fragile, cette frontière du Nord-Est et de l'Est est la plus remuante de toutes, la plus vivante car toujours en alerte, en raison du danger que constituent des voisins agressifs et redoutables. (...) Elle est pareille à toutes les frontières d'avant le XIXe siècle. Ne vous la représentez surtout pas comme une ligne à la moderne : la frontière linéaire, raide et abrupte (...) avec son double cordon de douaniers, ne date que d'hier. Jusque dans les traités de Louis $\mathrm{XV}$, on voit, par le grand nombre d'enclaves et d'exclaves qui font empiéter les Etats les uns sur les autres, que la conception de la frontière-ligne ne se dégage pas encore ». A Longwy, la frontière est figée en 1815 seulement, si bien que, plusieurs décennies plus tard, "c'est toujours la communauté villageoise et la paroisse [plutôt que le pays] qui fournissent aux habitants le sentiment d'appartenance à un groupe. » (Noiriel, 1984 : 34).

Probablement l'annexion de l'Alsace-Lorraine en 1871 a-t-elle eu plusieurs effets locaux conséquents. Le premier fut incontestablement une émulation industrielle de part et 
d'autre de la nouvelle frontière. Le découpage de 1871 ménageait côté allemand les champs ferrifères connus à l'époque, au nord de la Lorraine. Dès lors, l'Etat français a mis à disposition de l'Université de Nancy des moyens permettant d'en faire, selon les propos tenus par son recteur au tout début du $\mathrm{XX}^{\mathrm{e}}$ siècle, "une forteresse de la science où s'exerceront aussi de jeunes et vaillantes recrues pour l'armée industrielle de notre pays " (propos rapportés par G. Noiriel, 1984 : 77). C'est aussi ce « dynamisme revanchard » qui a stimulé la prospection de nouveaux champs ferrifères côté français, et les découvertes de Briey n'ont pas manqué de faire l'objet de propos patriotiques triomphants, tant dans les milieux politiques que dans les milieux d'affaires.

11 Le second effet de cette annexion concerne l'émergence de ce que l'on pourrait nommer un "effet frontière ». La III ${ }^{e}$ République exalta un très fort sentiment nationaliste, imposant de nouveaux symboles sur l'ensemble du territoire ${ }^{5}$. La Lorraine restée française occupa une place importante dans l'édification de ce patriotisme : «Les régions perdues sont au souvenir de chacun; quant à celles qui restent, elles acquièrent dans la communauté nationale une place singulière. Chacun les connaît, les respecte comme filles et rescapées du désastre. (...) Rapidement, la Lorraine française acquiert deux attributs spécifiques dans l'espace symbolique de la III République. Elle est à la fois un bastion et un phare, une vitrine de la France et de sa culture. » (Garcier, 2005 : 222). L'implantation massive de militaires et de forces de police - voilà pour le bastion - fait que, localement, "prestige naissant de l'uniforme, intérêt bien compris et haine du Prussien se conjuguent pour faire entrer dans les têtes le patriotisme» (Noiriel, 1984 : 60). Ainsi, peut-être pour la première fois de leur histoire avec une telle intensité, les Lorrains restés sur le sol national ont été érigés en véritables Français. Et si cette annexion d'une partie du territoire national s'est progressivement estompée des mémoires ${ }^{6}$, localement, son souvenir reste prégnant. A l'inverse, pour ceux qui ont été annexés, le doute s'est plus facilement instillép. Et de nos jours encore, le souvenir est vif de la frontière entre Moselle et Meurthe-et-Moselle, aujourd'hui départementale mais nationale plusieurs décennies durant.

12 Autre effet de lieu, l'industrialisation s'est faite par bassin, sur un mode globalisant. Chaque entreprise propriétaire de concession minière et/ou d'un appareil sidérurgique de transformation a plus ou moins rapidement «fixé " la main d'œuvre qu'elle embauchait. Rapidement aussi, la main-d'œuvre agricole locale convertie à l'industrie a manqué, et ce sont des travailleurs venus pour l'essentiel d'Italie et de Pologne que les usines et les mines ont embauché. Les industriels, exploitants miniers et maîtres de forge, ont bâti de véritables company-town à proximité du lieu de leurs productions, encadrant toute la vie "de la naissance à la mort ", selon leurs préceptes paternalistes ${ }^{8}$. Ainsi, s'est développé le sentiment d'une appartenance à une entreprise autant, sinon davantage, qu'à un lieu. C'est sans doute dans ce développement socio-spatial particulier qu'il faut chercher les marques encore prégnantes des trois bassins ferrifères lorrains, le Nord, autrefois annexé, et les Centre et Sud, chacun correspondant à des modes d'exploitation spécifiques comme à des histoires paternalistes singulières. Enfin, une autre conséquence de cette industrialisation au plus près des lieux d'extraction est que les villes lorraines pré-existantes, Nancy et plus encore Metz, ont longtemps été tenues à l'écart de ce développement économique. Pour le dire autrement, l'industrialisation lorraine n'a pas alimenté un phénomène urbain, mais plutôt une urbanisation diffuse autour d'anciens bourgs et villages.

1.3 Des règles et méthodes d'exploitation mouvantes 

façon générale, elle concerne en Lorraine des couches superficielles, situées entre 70 et 120 mètres maximum, c'est-à-dire beaucoup moins profondes que celles du bassin houiller situé plus à l'Est (entre 900 et $1.000 \mathrm{~m}$ ). La méthode d'exploitation s'organisait généralement en plusieurs phases. Dans un premier temps, étaient tracées des galeries principales rectilignes et de grande section ( 2 à $4 \mathrm{~m}$ environ), qui découpaient des «quartiers ». Dans chacun de ceux-ci étaient ensuite creusées des galeries secondaires, généralement obliques ou diagonales, délimitant des chantiers d'extraction très larges, parallèles. Les bandes laissées entre chacun d'entre eux étaient nommées "piliers ». Chacune de ces bandes était enfin coupée par des galeries tertiaires, ne laissant que des piliers résiduels de plus petite dimension. Ceux-ci étaient ensuite abattus, dans une opération appelée "dépilage » ou encore "torpillage », permettant ainsi de récupérer l'essentiel du minerai. A la suite de cette dernière opération, le toit de la galerie s'effondrait, et ce mouvement se poursuivait jusqu'à provoquer en surface un affaissement dit minier.

Une règle d'urbanisme avait établi que l'on ne pouvait construire sur des terrains dont les sous-sols dépassaient un «taux de défruitement » - soit le rapport de la partie extraite sur la partie restant dans le sous-sol - dépassant les $50 \%$. Cette règle valait aussi à l'inverse : on ne pouvait théoriquement exploiter à plus de $50 \%$ les veines dont le sol audessus était bâti. De même dans ces zones, on ne pouvait exploiter plus d'une couche dans l'épaisseur du sous-sol. Compte-tenu de la configuration urbaine évoquée plus haut, ces règles concernaient pour l'essentiel d'anciens villages et les cités ouvrières construites par les exploitants. Deux solutions étaient envisagées pour ces zones : soit on laissait les chambres et piliers intacts, soit on évitait d'exploiter les couches, créant ce qu'on appelait des «stots de protection ", c'est-à-dire des ceintures de $50 \mathrm{~m}$ non exploitées autour des zones urbanisées en surface, encadrées de murs de soutènement. A partir de 1976, et à la suite d'affaissements inquiétants observés en surface, le phénomène de fatigue des matériaux restants a été pris en compte dans la réglementation pour rapporter le taux de défruitement à $30 \%$ et non plus à $50 \%$ pour les exploitations sous les zones urbanisées. Autre élément d'ordre technique, lors de l'exploitation, les galeries pouvaient atteindre le niveau local de la nappe phréatique. Il fallait donc procéder de façon constante au pompage de ces eaux, opération appelée " exhaure », pour les rejeter dans les cours d'eau voisins ${ }^{9}$. A priori, lorsque l'exploitation s'arrête, l'exhaure est la dernière étape dans la procédure de fin de concession, car cela signifie qu'à terme, les galeries se remplissent d'eau. Incidemment, cette opération fragilise les chambres et piliers conservés sous les zones urbanisées ${ }^{10}$.

15 Nous verrons plus loin que le taux de défruitement constitue un indicateur important en matière d'effondrement minier. Or certaines mines ont connu des surexploitations, donc des taux dépassant ceux théoriquement autorisés. On cite couramment les deux périodes de guerre mondiale pendant lesquelles les demandes en minerai étaient plus abondantes, d'un côté comme de l'autre de la frontière, et où l'observation de ces règles s'est semble-til relâchée. On évoque aussi le cas des mines dites «marchandes ", c'est-à-dire non directement intégrées à un système d'exploitation sidérurgique aval. Leur structure économique isolée rendait leurs actionnaires plus fragiles, et les exploitants pouvaient être tentés de rechercher une plus grande rentabilité quitte à enfreindre les règles. Autre cas rencontré, lorsqu'un exploitant trouvait une veine particulièrement riche, c'est-à-dire dépassant la faible teneur habituelle en fer, il pouvait être aussi tenté de l'exploiter à 
l'excès. On trouve aujourd'hui plusieurs cas de ce type, à Auboué, par exemple, où des cités minières ont été bâties sur des terrains exploités à des taux supérieurs à $70 \%$, ou encore à Nonkeil-ottange, dans le bassin Nord, où trois galeries ont été exploitées les unes sur les autres.

Enfin, ultime élément de complexification, au fil du siècle de l'exploitation de ces mines, les entreprises concessionnaires ont fait l'objet de rachats, de regroupements ou autres mouvements liés à ceux des capitaux dont elles dépendaient. Une même mine aura donc pu être exploitée d'une manière peu scrupuleuse à une certaine époque et de façon totalement conforme à une autre, et inversement. Ainsi peut-on dire que, tout le temps de la période de son exploitation, le sous-sol a "travaillé » dans un cadre réglementaire mouvant, et selon des cadres de production différents. C'est donc peu dire que, au moment de l'arrêt des exploitations, tous les changements évoqués - connus ou non de l'administration, engendrés ou non par elle - rendent l'état réel des lieux particulièrement complexe à établir. Et une fois l'extraction stoppée, le sous-sol a continué de travailler de façon accélérée avec l'ennoyage des bassins.

\subsection{Une désindustrialisation brutale et douloureuse}

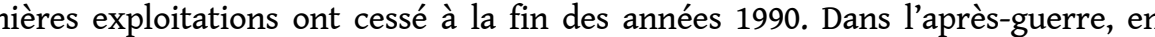
effet, les usines sidérurgiques produisirent au maximum de leur capacité, dans cette période de reconstruction du pays où le fer - et le charbon - ont joué un rôle primordial. La Lorraine produisait alors les trois quarts de la fonte et les deux tiers de l'acier en France avec près de 100.000 sidérurgistes et 30.000 mineurs de fer. En particulier, le système industriel intégré du bassin Sud, autour des installations de Joeuf, a connu son apogée dans les années 1960 et 1970. Puis survinrent les années de crise, à partir de 1970. On les explique généralement, non tant par un épuisement local de la ressource ferrifère, mais davantage par un faible coût des matières premières provenant de pays du Sud, plus riches en fer. La baisse des coûts du transport dans le courant des années $1960^{11}$ en a ajouté pour favoriser un changement de fournisseur. Enfin les maîtres de forge lorrains ont globalement peu modernisé leur appareil productif pendant plusieurs décennies, le choc pétrolier de 1973 venant surenchérir le coût d'exploitation d'installations vieillissantes. Entre 1975 et 1994, l'emploi industriel lorrain chute de 144.000 emplois. Depuis, la sidérurgie locale a quasiment disparu, en vagues successives, dans le courant des années 1980 et 1990. Les mines du bassin Sud ont commencé à fermer en 1964 (autour de Trieux), la dernière date de 1992. Aujourd'hui, aucune mine de fer n'est plus exploitée en Lorraine : la dernière a été fermée en juillet 1998, à Audun-le-Tiche, dans le bassin Nord. Et encore était-elle exploitée depuis le Luxembourg.

Les fermetures de mines et d'usines ont aussi marqué la fin d'un "système industriel total », dans lequel l'employeur prenait en charge non seulement le travail, mais aussi quantité d'autres aspects de la vie (l'habitat, la santé, les sports, la culture et les loisirs, etc.). La solidité de ce système était fondée sur une forme de garantie de l'emploi sur plusieurs générations et une relative protection sociale pour les salariés et leur famille. Les fermetures ont localement représenté bien plus que la seule perte de l'emploi. Or au contexte catastrophique ambiant, vécu comme tel par de nombreux Lorrains, sont venus s'ajouter d'autres sinistres. En effet, la fin progressive des exploitations s'est accompagnée de plusieurs effondrements d'anciens quartiers miniers.

2. Un risque minier érigé en lutte sociale

On l'aura compris, un affaissement minier dépend, pour l'essentiel, de la nature du soussol et de sa composition, de la profondeur de l'exploitation minière, de la façon dont 
celle-ci a été conduite (et en particulier du taux de défruitement) et enfin de l'effet de l'eau lorsque les galeries ne sont plus exploitées ni exhaurées. Sa manifestation revêt diverses formes, mais les plus courantes, en fragilisant fortement les structures porteuses, rendent inutilisables les bâtiments affectés.

2.1 Logement minier, la clause qui fâche

Il faut préciser tout d'abord les conditions particulières du logement minier. En effet, le statut du mineur a été décrété en 1946 au lendemain d'une guerre qui demandait à cette corporation un effort particulier dans la reconstruction du pays. En forme de contrepartie, le gouvernement issu du Conseil national de la Résistance a institué dans son article 23 le droit au logement gratuit pour les mineurs, actifs comme retraités, ainsi que pour leurs veuves considérées comme ayant-droit ${ }^{12}$. Autrement dit, les compagnies étaient propriétaires de maisons dont elles ne tiraient plus aucun bénéfice, devenues en quelque sorte un appendice de leur appareil de production.

Dans la période de désindustrialisation, les compagnies minières et sidérurgiques se sont progressivement dessaisies de leur patrimoine immobilier, peu entretenu les dernières années, en le vendant à ses occupants pour un coût relativement modique. Mais dans les contrats de vente, au moins jusqu'en 1994, les propriétaires-exploitants avaient généralement ajouté une clause exonérant leur responsabilité en cas de désordres liés à des mouvements de sol et de sous-sol.

Des affaissements miniers, il en exista pendant la période d'extraction. L'un des plus spectaculaires fut peut-être celui de Moutiers (54), en 1940, où quarante quatrelogements s'effondrèrent brutalement en pleine nuit ${ }^{13}$. Dans ce cas comme dans d'autres, il s'agissait d'une affaire industrielle interne, dont la gestion relevait du seul exploitant: les sinistrés étaient relogés par la compagnie, qui réparait les maisons lorsque c'était possible ou qui les détruisait et en reconstruisait d'autres le cas échéant. Mais la perspective s'est modifiée avec la vente des cités à leurs habitants. Lors des premiers affaissements des maisons " clausées ", les sinistrés ont eu à faire face à un vide juridique en termes de responsabilité.

2.2 Premiers affaissements dans les bassins Sud et Centre

Les premiers affaissements de ce type ont eu lieu dans les bassins Sud et Centre, qui avaient été ennoyés au début des années 1990. En octobre 1996 a eu lieu un affaissement minier dans la cité de Coinville, à Auboué (54), touchant environ quatre-vingt maisons et les rendant inhabitables (toits affaissés, trous béants dans les voiries, etc.). En novembre de la même année, toujours à Auboué, rue de Metz, à nouveau quatre-vingt maisons s'affaissent. En mai 1997, un affaissement se produit à Moutiers (54), pendant une visite préfectorale. En 1998, plusieurs autres quartiers sont touchés à Moyeuvre (57), à Montoisla-Montagne (57) et à Roncours (57). Au début des années 2000, on estimait à environ cinq cents le nombre de maisons touchées dans le bassin ferrifère, représentant près de deux mille habitants évacués, pour l'essentiel des propriétaires occupants expropriés.

Il s'agit généralement de populations minières fragiles parce qu'âgées, souvent issues d'une immigration récente, maghrébine ou turque, ou plus ancienne, italienne ou polonaise, et pour qui un relogement en immeuble, de façon éclatée au gré des disponibilités dans le parc HLM, représente une profonde rupture avec ses pratiques antérieures. L'obligation d'évacuer leur espace intime, et de quitter une communauté construite autour du travail, constituait un traumatisme au moins aussi important que ne l'avait été en son temps l'annonce de l'arrêt de toute activité minière ${ }^{14}$. Face au discret 
effacement des responsables, les sinistrés «clausés » vont se retourner vers les pouvoirs publics, érigeant ainsi ce problème en une " affaire d'Etat».

En effet, lorsqu'un exploitant minier stoppe une production, il doit se conformer à une longue procédure dite de fin d'exploitation avant de rendre à l'Etat la concession du soussol qui lui avait été octroyée. Cette procédure a pour objet en particulier d'effectuer une sorte d'état des lieux du sous-sol, à la manière d'un locataire quittant son appartement. Généralement, la fin de l'exhaure est prononcée en phase finale du processus, qui s'achève par la promulgation d'un arrêté ministériel au terme duquel l'exploitant n'est plus propriétaire de la concession. A ce titre, il n'est définitivement plus responsable des dégâts ultérieurs éventuels causés par des désordres du sous-sol ${ }^{15}$. Lorsque survinrent les premiers affaissements lorrains, la procédure de fermeture des bassins Centre et Sud était quasiment achevée, ne manquait plus que l'arrêté ministériel. Ainsi les sinistrés se sontils trouvés dans une impasse juridique: ils ne pouvaient se retourner ni contre un exploitant qui, avec les "clauses", s'estimait dégagé de ses responsabilités, ni contre l'Etat jugé non encore responsable de ces affaissements puisque la renonciation à la concession n'était pas totalement achevée. Une première loi avait été votée en $1994^{16}$, afin de déclarer la nullité d'ordre public des clauses minières sur la non-responsabilité des compagnies dans les désordres des sous-sols. Mais cette disposition n'est pas rétroactive ${ }^{17}$ , et l'essentiel des cités minières avaient été vendues avant 1994.

\subsection{L'émergence d'un collectif des communes minières}

En mars 1997, à la suite des affaissements d'Auboué, un Collectif des communes minières de Lorraine s'est créé, à l'initiative de Colette Goeuriot, députée-maire (Apparentée PCF) de Joeuf ${ }^{18}$. Trois principaux objectifs l'animent. Il s'agissait tout d'abord d'obtenir une indemnisation rapide des sinistrés, avec en toile de fond le vote d'une législation garantissant et encadrant ces réparations. Le second point concernait l'obtention de moyens financiers et réglementaires pour les communes en proie aux risques d'affaissements miniers, dans un contexte de reconversion économique particulièrement crucial. Enfin le Collectif désignait assez clairement l'ennoyage des bassins Sud et Centre comme phénomène accélérateur des affaissements. Dans cette optique, il conjurait les pouvoirs publics de ne pas ennoyer le bassin Nord et d'y pratiquer les consolidations nécessaires dans les zones qui seraient désignées à risques.

Trois collèges composent ce Collectif. Le premier regroupe les communes minières et très rapidement, une centaine vont se porter adhérentes (dont certaines du bassin houiller voisin). Le second rassemble des associations, en particulier les associations locales de sinistrés, ainsi que la Fédération CGT des mineurs, organisation syndicale de tout temps majoritaire dans la corporation. Enfin on trouve dans le dernier collège des personnalités qualifiées pour leur intérêt et leur compétence sur la question. Il s'agit au final d'un Collectif large, mais dont l'épicentre se situe assez clairement à la gauche de l'échiquier politique, compte tenu de son ancrage territorial dans d'anciens bassins miniers. D'une certaine manière, il va permettre à un grand nombre d'acteurs politiques, syndicaux et associatifs essentiellement issus du monde social de la mine en désagrégation, de se remobiliser autour d'une actualité touchant à la fois au passé minier et à l'avenir menacé de ces territoires. Il va faire pression de façon incessante sur le pouvoir central et sur les administrations pour obtenir une amélioration de la situation.

En 1996, 1997 et 1998, les premiers sinistrés « clausés » ont pu être indemnisés, sur la base d'accords amiables passés entre l'Etat, l'ancien exploitant minier ${ }^{19}$ et son assurance ${ }^{20}$. Le rapport de force et l'émotion suscitée aidant, les indemnisations sont généralement 
qualifiées de " correctes " par les indemnisés (de 600 à $800 \mathrm{KF}$, soit 91 à $122 \mathrm{~K} €$ ). Elles ont été établies non sur la base de la valeur vénale de la maison, mais sur celle de la somme nécessaire pour en racheter une autre, sur « la valeur de reconstruction à neuf ». Mais il s'agit là d'accords au cas par cas, en rien généralisables.

2.4 L'Etat reprend la main

Après un travail tantôt revendicatif tantôt constructif du Collectif et de toutes les organisations qui le soutenaient, les parlementaires lorrains ${ }^{21}$ obtiennent en 1999 le vote d'une loi $^{22}$ relative à l'indemnisation des victimes ${ }^{23}$. Partant de ce texte, l'exploitant est désormais considéré comme responsable des désordres du sous-sol, mais c'est l'Etat qui prend en charge l'indemnité des victimes. Une légère rétroactivité permet de couvrir une partie des cas en cours, toutefois les sinistres datant d'avant le $1^{\text {er }}$ septembre 1998 ne sont toujours pas pris en compte. Ils se solderont au cas par cas. En ce sens, il s'agit davantage d'une loi préventive anticipant de probables futurs affaissements. Autre point important, ce texte promulgue les Plans de prévention des risques miniers (PPRM), qui permettent des expropriations préventives, octroient la possibilité de consolider certains sous-sols, et surtout réglementent à terme le droit des sols sur les zones considérées comme dangereuses.

Paradoxalement, c'est sur ce second point que les réactions des communes du Collectif vont être les plus vives. Car, alors qu'elles demandaient des moyens supplémentaires pour appuyer leur reconversion, les services de l'Etat, appliquant un principe de précaution, gelèrent toute urbanisation nouvelle dans les communes minières, en attendant la promulgation des futurs PPRM dont il est annoncé qu'elle pourra prendre jusqu'à dix années. Certaines étant déjà dans une situation de sinistre économique avancée, elles interprétèrent cette durée comme un coup de grâce final.

\section{Le PPRM : une réponse techniciste de l'Etat}

31 Dès lors, l'élaboration et la mise en œuvre des PPRM va se passer dans un contexte de très forte tension entre les collectivités locales et les services de l'Etat, la Direction régionale de l'industrie, la recherche et l'environnement (DRIRE) première concernée et, dans une moindre mesure, la Direction régionale de l'équipement (DRE). Il s'agit d'une situation somme toute assez banale s'agissant de négociations de plans de prévention ${ }^{24}$. L'élément nouveau consiste ici en la mobilisation du Collectif qui, en recyclant des modes d'action forgés du temps de la mine, a exercé une pression constante sur l'administration à toutes les étapes de la négociation, tout en popularisant régulièrement ses luttes. Un des modes de défense de cette dernière a visiblement été de tenter de «techniciser » au plus le débat.

3.1 L'élaboration « minée » d'une cartographie des risques

Une des premières réponses de l'Etat aux affaissements de 1996 et de 1997 avait été de geler unilatéralement l'urbanisme dans les anciennes communes minières, en attendant des mesures de prévention. En parallèle, les ministères de l'Environnement et de l'Industrie avaient apporté une aide à la DRIRE de Lorraine pour mieux comprendre les phénomènes redoutés et pour pouvoir établir au plus vite une cartographie des risques. Dans cette dynamique, avaient été créés le GISOS ${ }^{25}$, pour étudier les comportement des terrains et les impacts en surface, puis GEODERIS ${ }^{26}$ pour appliquer ces recherches au travers de systèmes d'information géographiques miniers, de modes de surveillance, de traitement et d'analyse des risques, voire même de suivis techniques de chantiers. Cette expertise de l'Etat a permis de produire une définition "réglementaire " claire des 
phénomènes étudiés, en distinguant trois types de risques - l'affaissement ${ }^{27}$, le fontis ${ }^{28}$ et l'affaissement brutal ${ }^{29}$ - auxquels correspondraient des modes de traitement différenciés.

Les PPRM ont été élaborés à partir de $2000^{30}$. Leur obligation de production a été confirmée localement par la «Directive territoriale d'aménagement Lorraine ${ }^{31}$ » qui va définir une doctrine administrative en matière de réglementation de la constructibilité dans les zones de risques miniers. Celle-ci établit un arbitrage entre la sécurité et le développement de communes touchées par l'après-mine. Comme tous les plans de prévention, les PPRM $^{32}$ sont prescrits par l'Etat, élaborés par les services de l'Etat (ici DRIRE et DDE), s'imposent ensuite aux communes et doivent être intégrés dans les PLU. Leur principe repose sur deux piliers. Il s'agit d'une part, pour la DRIRE, de définir les dangers que recèle le sous-sol, dans une classification établie en fonction de la connaissance que l'on a des phénomènes d'affaissement, de la localisation des galeries et du degré d'exploitation des mines. Il s'agit ensuite, pour la DRIRE et la DRE, de transposer ces dangers pour les sols (urbanisés ou non), à travers une cartographie qui puisse rendre compte de la nature et du degré de risques à l'échelle de chaque parcelle.

Nous n'entrerons pas ici dans le détail de la fabrication de ces PPRM $^{33}$. Retenons pour l'essentiel qu'il s'est agi d'une "réglementation dynamique ", en évolution au fur et à mesure des capacités qu'ont eu les scientifiques mobilisés pour l'occasion à sérier et à caractériser les phénomènes ${ }^{34}$. Plusieurs générations de zonages ont été élaborées, les premières temporaires sans nécessairement qu'elles soient présentées comme telles. La dynamique est aussi venue de la pression permanente exercée par le Collectif et toutes les forces sociales mobilisées, modifiant sensiblement les modes de travail habituels des agents de l'Etat ${ }^{35}$.

La confrontation s'est cristallisée en particulier autour de l'élaboration de la cartographie des aléas. En effet, la définition des dangers que recèlent les sous-sols tient, on l'aura compris, à la connaissance que l'on a de leur état. Or deux des trois bassins avaient déjà été ennoyé, et il était donc devenu impossible d'aller y vérifier l'état réel du sous-sol. Quant au bassin Nord, encore exhauré pendant toute cette période d'élaboration, vérifier de visu l'état des galeries s'avérait hasardeux : certaines salles étaient effondrées, ou bien encore certains barrages souterrains n'étaient plus entretenus et avaient conduit à l'ennoyage de telle ou telle galerie, etc. Bref, les études d'aléas ont été réalisées en grande partie par extrapolation de documents anciens et non d'après des relevés actualisés. Et la mise au jour de ces connaissances, de la responsabilité de la DRIRE, fut problématique.

3.2 La cartographie des aléas au cœur des contestations

Les contestations se sont donc beaucoup concentrées sur la production de la cartographie des aléas. Tout d'abord, les études d'aléas miniers ont été réalisées par la DRIRE à partir des dossiers d'arrêts des exploitants. En effet, dans chaque exploitation active, un géomètre était chargé d'établir et de mettre à jour une cartographie du sous-sol, au fur et à mesure de l'avancée du chantier. Cette cartographie permettait le dialogue avec le service des Mines (ancêtre des DRIRE), en charge de la police des mines, mais permettait aussi au chef de chantier de rendre compte à l'exploitant de l'avancement du travail. Il s'agit donc de documents au statut ambigu, poursuivant deux finalités parfois contradictoires, en terme de respect des règles d'exploitation (taux de défruitement, largeur des piliers, non superposition des galeries, etc.), et de rendement du chantier. Ainsi les documents récoltés, variant selon les époques, pouvaient accentuer l'un ou l'autre aspect sans nécessairement renseigner sur une "vérité vraie » de l'état réel du sous-sol. 
37 Par ailleurs, les concessions ferrifères ont été élaborées il y a plus d'un siècle. Se sont côtoyées de petites usines sidérurgiques, qui elles-mêmes avaient acheté de petites concessions, comme de gros complexes industriels. Lormines, entreprise qui au final a rendu les concessions Sud et Centre à l'Etat, s'était constituée sur tout un conglomérat de petites et grandes affaires totalement hétéroclites, disposant les unes de documents d'archive réguliers, d'autres de presque aucun fonds cartographique. De plus, il pouvait exister des blancs plus ou moins importants concernant les période d'annexion allemande, et plus précisément les périodes de guerre. Cet ensemble composite était connu comme tel par les principaux acteurs du Collectif. Le récolement des cartes, particulièrement complexe à effectuer, a donné lieu à de multiples contestations locales. Le responsable d'une association de sinistrés nous déclarait : «On sait que la cartographie remise [en mairie par la DRIRE et la DDE] est fausse, par recoupement avec l'expérience des mineurs".

Enfin, autre point régulièrement soulevé par les membres du Collectif, il existait parfois une complicité non dissimulée entre l'ancien service des Mines et les exploitants et chefs de chantiers. Un responsable d'association de sinistrés nous a confié : "Certains inspecteurs, c'était de notoriété publique chez les mineurs, rencontraient le chef de chantier dans le café jouxtant le puits, et ne descendaient jamais avec eux au fond pour vérifier quoi que ce soit. Du coup, les documents remis par l'exploitant pouvaient être faux ou truqués ». On perçoit là que les oppositions et contestations autour de l'élaboration de ces documents de planification ont été nourries côté collectivités locales de cadres d'analyse directement héritées des pratiques et des modes de faire «du temps de la mine». Au fond, deux des principaux protagonistes - anciens mineurs et ancien service des mines - étaient encore présents dans ce conflit. A ceci près que la DRIRE, héritière du Service des mines mais composée d'une génération d'ingénieurs n'ayant jamais connu la période d'exploitation, a souhaité mettre le plus à distance possible cette histoire sociale et ses ramifications, en s'en tenant à quelques éléments de la seule histoire technique.

Conclusion

39 Au final, l'ennoyage du bassin Nord, qui correspond au stade ultime de la procédure de fin d'exploitation, représentait probablement pour les agents des services de l'Etat un moyen d'en finir définitivement, matériellement comme symboliquement, avec le temps de la mine. Avec les galeries, peut-être espéraient-ils aussi noyer l'histoire sociale et politique de la mine, tout du moins en atténuer ses réminiscences contemporaines. "L'ennoyage, ça va nous permettre enfin de tourner la page ", nous confiait l'un d'eux.

Et effectivement, lors de ces affaissements miniers et de leurs suites réglementaires, se sont joués des chocs de temporalité. Des tensions sont nées de l'urgence dans laquelle se sont retrouvés des sinistrés, qui souhaitaient retrouver au plus vite une situation à l'identique, confrontés aux lenteurs administratives nées du comblement complexe d'un vide juridique sur ce problème. S'est jouée aussi parfois la réactivation d'une mémoire encore présente des «Français du dehors", qui se sont sentis comme une nouvelle fois «abandonnés » par l'Etat, dans un contexte de décentralisation dans lequel la mission de reconversion industrielle étant partiellement transférée aux collectivités territoriales. Se sont confrontés, enfin, deux représentations différenciées du danger d'affaissement. L'une, guidée par la nécessité d'agir, a été produite par des agents et des organisations habitués à raisonner dans une logique sectorielle bornée pour l'essentiel par le problème à résoudre et ce dans la limite des moyens matériels et humains disponibles. L'autre, à l'inverse, enracinée dans l'expérience individuelle et collective du territoire, s'inscrivait 
dans le temps long et dans le souvenir de la confrontation à un exploitant présent dans quasiment toutes les pratiques de la vie quotidienne.

Cela étant, c'est précisément parce qu'il y avait un contexte territorial spécifique, avec la mobilisation de forces sociales encore chargées d'une histoire toute proche, qu'une dimension politique s'est imposée dans la gestion et le traitement de ces risques. Plusieurs thèmes, relevant de choix politiques, ont pu traverser les débats sur les affaissements. L'un d'eux concerne le rôle de l'Etat, passé et présent, vis-à-vis de territoires en crise, et l'existence ou non d'une autorité solidaire et régulatrice qui pallie les moyens des collectivités territoriales lorsqu'ils s'avèrent faibles. Un autre tient à la façon dont on fixe les limites de la responsabilité des entreprises minières dans la dégradation des sous-sols qu'elles ont exploités. Un autre encore, non évoqué ici, a concerné la possibilité de poursuivre l'exhaure afin de produire une eau publique de consommation, en transférant symboliquement aux collectivités locales le système de pompage existant.

Enfin, un dernier thème, et non des moindres, a concerné le (non) partage de l'information. Les services de l'Etat parlent volontiers de "laboratoire Lorrain», pour décrire cette décennie 1995 - 2005 nécessaire à la compréhension des phénomènes mécaniques d'affaissement et à la mise en règlement des PPRM. Cette expression renvoie bien à une dimension expérimentale et locale, éclairante pour d'autres situations nationales. Or s'il s'est bien agi d'une expérimentation de l'Etat local, menée avec e renfort d'une expertise d'Etat venant de divers horizons, elle a été menée en grande partie en étanchéité avec les associations et les collectivités constituant l'expression provenant du territoire. Pourtant, l'exploitation minière avait été capable par le passé d'inventer des formes de co-gestion sur la sécurité, par exemple avec l'élection de délégués mineurs dès 1910. Dans la continuité de cette histoire, associations et collectivités, réunies dans le Collectif, ont été en capacité d'apporter une expertise qui aurait pu enrichir les documents produits, voire parfois modifier les solutions apportées.

C'est peut-être cette confrontation politique autour de la gestion du risque, redécouverte dans le contexte lorrain, qui constitue le véritable « laboratoire Lorrain des risques ». Car elle redonne quelques clés de lecture à d'autres situations locales de mises en œuvre de plans de prévention de risques, dans lesquelles la Technique, généralement mise en avant par les agents des services de l'Etat, masque ou aseptise des situations conflictuelles rendues de ce fait illisibles et invisibles.

\section{BIBLIOGRAPHIE}

Baudelle Guy, 1996, « La gestion des risques hérités de l'activité minière dans le Nord-Pas-deCalais », Revue de géographie de Lyon, volume 71-1, p. 65-70.

Bayet Cyril et Le Bourhis Jean-Pierre, 2000, « Le zonage comme instrument de gouvernement. Le cas des risques naturels », Annales des Ponts et Chaussées, n93, p. 52-58. 
Blanchard Pascal et Lemaire Sandrine, 2003, Culture coloniale. La France conquise par son Empire. 1871-1931, Editions Autrement, collection Mémoires, 253 p.

Braudel Fernand, 1986 (1990), L'identité de la France. Espace et histoire, Flammarion, collection Champs, Paris, $410 \mathrm{p}$.

Coanus Thierry, 2006, « Approcher la notion de risque en sciences sociales : réflexions liminaires », in Coanus T. et Pérouse J.-F., Villes et risques. Regards croisés sur quelques cités «en danger ", Economica Anthropos, Paris, 245 p.

Dedeler Virginie et Tarquinio Cyril, 2004, « Impacts psychosociaux des risques d'affaissements miniers. Anxiété, perception de l'environnement et accès à l'information », Annales des mines, juillet 2004, p. 45-50.

Di MéoGuy, 1998, Géographie sociale et territoires, Editions Nathan université, Collection Fac. géographie, Paris, $320 \mathrm{p}$.

Duchêne François, 2001, «Quand le risque se heurte au territoire : la révision du Plan d'occupation des sols autour d'une usine chimique ", Colloque international Risques et territoires, UMR-CNRS 5600 Environnement, Ville, Société, 16-18 mai 2001, ENTPE, Vaulx-en-Velin, Tome 2, p. 109-126.

Duchêne François (dir.) et Morel Journel Christelle (dir.), Coanus Thierry, Martinais Emmanuel, 2004, De la culture du risque. Paroles riveraines à propos de deux cours d'eau périurbains, Éditions de l'Aube, collection Recherche, Société et territoire, $170 \mathrm{p}$.

Filippetti Aurélie, 2003, Les derniers jours de la classe ouvrière, Stock, Paris, 189 p

Frey Jean Pierre, 1986, La ville industrielle et ses urbanités. La distinction ouvriers / employés au Creusot, 1870 - 1930, Pierre Mardaga éditeur, Liège, 386 p.

Frey Jean Pierre, 1995, Le rôle social du patronat. Du paternalisme à l'urbanisme, L'Harmattan, Paris, $383 \mathrm{p}$.

Garcier Romain, 2005, La pollution industrielle de la Moselle française. Naissance, développement et gestion d'un problème environnemental. 1850-2000, Doctorat de Géographie, Direction J. P. Bravard, Université Lyon 2, $487 \mathrm{p}$.

Lamotte Guy, « Des mines de fer de Lorraine aux mines de la vallée de l'Orne », nr.

Martinais Emmanuel, 1996, « Gestion du risque industriel et conflits territoriaux - Le cas de StFons, commune de l'agglomération lyonnaise ", Revue de géographie de Lyon, vol. 71.1/96, p. 31-44.

Martinais Emmanuel, 2007, La mise en règlement des Plans de prévention des risques technologiques (PPRT). Production normative et réforme de la prévention des risques industriels, Programme de recherche « Risque Décision Territoire » du ministère de l'Ecologie et du développement durable, $2^{\mathrm{e}}$ rapport du projet de recherche « les territoires du risque », dir. E. Martinais, 104 p.

Chaut- Morel Journel Christelle, 1996, « Un « nouvel » acteur paradoxal en région stéphanoise : les Houillères de bassin du Centre et du Midi », Revue de géographie de Lyon, volume 71-3, p. 209-215.

Morel Journel Christelle, 2007, « De la maison ouvrière au pavillon : quand les « signes des temps miniers » s'estompent à la Cité des Combes ", In Cités ouvrières en devenir, F. Duchêne (dir.), à paraitre.

Noiriel Gérard, 1984, Longwy, immigrés et prolétaires. 1880-1980, PUF, Paris, 396 p.

Rondeau Daniel et Baudin François, 1979, Chagrin Lorrain. La vie ouvrière en Lorraine (1870-1914), Editions du Seuil, Paris, 145 p. 
Veltz Pierre, 2000, Le nouveau monde industriel, Ed. Gallimard, Coll. Le débat, Paris, 230 p.

\section{NOTES}

1. En janvier 2005 et février 2006, dans le cadre du module « Risques et sociétés » du Mastère Gestion des risques de l'ENTPE.

2. Nous avons rencontré des agents de la DRIRE et de la DRE Lorraine, un dirigeant de la société Lormines, de nombreux élus et parlementaires locaux, plusieurs responsables d'associations de sinistrés, un représentant de compagnie d'assurance, des habitants directement touchés par un affaissement, et nous sommes rendus sur plusieurs sites sinistrés ou menacés de l'être.

3. Nous retiendrons avec Guy Di Méo l'idée que le territoire, pris entre « l'ordre du pouvoir » et « l'ordre de la nature », est avant tout une construction sociale. Il « reflète un type de rapport à l'espace propre à plusieurs individus, à un groupe ou à plusieurs groupes. Rapport spatial doté de l'aptitude de modifier, de médiatiser en retour l'ensemble des relations sociales qui lui ont donné naissance » (Di Méo, 1998 : 273).

4. 28 à $34 \%$ de teneur en fer seulement, d'où ce nom diminutif.

5. L'école de Jules Ferry obligatoire, mais aussi la Marseillaise qui devient hymne officiel en 1879 , et le 14 juillet proclamé fête nationale en 1880 .

6. On a pu oublier aujourd'hui l'ampleur du traumatisme. Dans leurs travaux sur la diffusion de la culture colonialiste, contemporaine de l'annexion de l'Alsace-Lorraine, P. Blanchard et S. Lemaire (2003 : 29) rappellent ces faits : « La célèbre réponse de Déroulède à Ferry [à l'Assemblée nationale] synthétise parfaitement la position de la droite nationaliste : «J'ai perdu deux enfants [l'Alsace et la Lorraine] et vous m'offrez vingt domestiques [les vingt pays de l'Empire colonial]». La gauche républicaine n'est pas en reste et s'inscrit dans la même perspective, comme l'écrit Juliette Adam : " Chaque pelletée de terre coloniale me paraît une pelletée de terre rejetée de l'Alsace-Lorraine en Prusse ». Ces oppositions [à la conquête coloniale] découlent de motifs hétérogènes et pourtant proches : pour la droite conservatrice et une partie de la gauche, la colonisation participe à la dilution de la puissance française, alors que toutes ses forces devraient se concentrer sur la reconquête de l'Alsace et de la Lorraine ».

7. Dans un roman mettant en scène l'histoire d'Audun-le-Tiche, sa ville minière natale autrefois allemande, Aurélie Filippetti (2003) donne à voir par touches les conséquences de ces annexions pour ceux qui se sont nommés plus tard « les Français du dehors ». 8. Sur ce point, voir par exemple l'ouvrage de J.P. Frey (1986), montrant la projection par les maîtres de forge du Creusot, la famille Schneider, des rapports sociaux de l'usine sur l'espace urbanisé ; voir aussi, du même auteur, les origines du patronage et du paternalisme (1995).

9. Les eaux d'exhaure constituent une ressource importante en eau potable, que certains exploitants miniers ont partiellement rentabilisé.

10. Toutes ces informations d'ordre technique nous ont été délivrées par d'anciens cadres miniers, des élus locaux et des personnels de la DRIRE. Elles ont été recoupées autant que possible à l'aide de diverses sources techniques.

11. Plus précisément la réduction des coûts de transport (maritime en particulier) concomitante à l'abaissement général des barrières tarifaires et non tarifaires limitant les échanges (Veltz, 2000 : 97). 
12. Ceux qui ne sont pas logés directement par leur compagnie perçoivent de la part de celle-ci une indemnité de compensation. Sur ces point, voir par exemple Chaut - Morel Journel (1996) et Morel-Journel (2007).

13. Voir l'article de l'historien local Guy Lamotte « Des mines de fer de Lorraine aux mines de la vallée de l'Orne », non daté et non référencé.

14. Sur ce point, voir l'étude des psychosociologues V. Dedeler et C. Tarquinio (2004). 15. La question de la durée des responsabilités des affaissements fait débat. Dans les bassins miniers du Nord, exploités plus profondément que ceux ferrifères lorrains, certains estiment que les affaissements interviennent très rapidement après l'exploitation, d'autres constatent que le phénomène peut avoir lieu jusqu'à vingt années plus tard (Baudelle, 1996, 67).

16. Loi $\mathrm{n}^{\circ} 94-588$ du 15 juillet 1994 , modifiant le Code minier et le Code du travail.

17. Ce qui sera confirmé par un avis du Conseil d'Etat du 23 septembre 1997.

18. La commune d'Auboué se situe dans sa circonscription.

19. Lormines pour les bassins Centre et Sud, actionnaire d'Usinor et qui n'existe plus en tant qu'exploitant.

20. AXA unique assureur.

21. A cette occasion, Jean-Yves Le Deaut, député PS de Meurthe-et-Moselle, parlait même de « mini parlement Lorrain » pour souligner l'entente entre les parlementaires lorrains, au-delà de leurs divergences politiques, pour porter ce texte et le faire aboutir.

22. Loi n99-245 du 30 mars 1999.

23. La loi Bachelot du 30 juillet 2003 inclura aussi des dispositions sur les risques miniers, en particulier en matière de délais d'indemnisation.

24. Sur ce point, voir par exemple Martinais (1996), Duchêne (2001), ou Bayet et Le Bourhis (2000).

25. Groupe d'intérêt scientifique sur l'impact et la sécurité des ouvrages souterrains, regroupant le BRGM, l'INERIS, les Ecoles des mines de Paris et Nancy et l'Ecole de géologie de Nantes.

26. Regroupant le BRGM et l'INERIS.

27. Il correspond à une instabilité qui peut survenir au-dessus d'une exploitation par chambre et piliers, et se traduit par la formation en surface d'une cuvette de quelques dizaines à quelques centaines de mètres de diamètre. L'affaissement de la surface se produit généralement progressivement en quelques jours ou en quelques mois.

28. Il s'agit de l'apparition soudaine en surface d'un entonnoir de quelques mètres de rayon et quelques mètres de profondeur, qui fait suite à une dégradation progressive de la voûte d'une galerie et qui remonte peu à peu dans le recouvrement jusqu'à percer au jour.

29. Dans certains cas, la ruine de l'édifice minier ne se fait pas progressivement mais on observe l'effondrement en bloc de l'ensemble des terrains compris entre le fonds et la surface. L'image qui prévaut est celle d'une table dont les pieds se dérobent subitement. L'effondrement de la surface se produit alors de manière dynamique, en quelques secondes. Une forte secousse sismique est ressentie.

30. Le décret d'application de la loi de 1999 date du 16 juin 2000.

31. Document indiquant les priorités de l'Etat sur un territoire assez large, qui s'impose aux Schémas de cohérence territoriale (SCOT) et aux Plans locaux d'urbanisme (PLU). 32. Il sont composés d'un document de présentation, d'un plan de zonage, indiquant les secteurs affectés par le risque (certains secteurs deviennent non constructibles à l'urbanisation future, d'autres restent constructibles, mais avec des prescriptions 
particulières touchant essentiellement au type de bâtiments autorisés et aux normes de construction spécifiques pour ces bâtiments) et d'un règlement précisant ces normes de construction.

33. Nous ne disposons pas des éléments nécessaires pour le faire. Le travail d'Emmanuel Martinais (2007) sur l'élaboration des PPRT montre qu'il s'agit bien d'une recherche à part entière.

34. L'affaissement brutal, par exemple, phénomène le plus redouté par les services de l'Etat car il touche directement à la sécurité des personnes, a été identifié plus tardivement, vers 2000.

35. Le refus d'instaurer une transparence autour de leur travail a été l'un des points les plus reprochés par les collectivités locales aux administrations centrales.

\section{RÉSUMÉS}

Avec la fin de l'extraction et la vente des cités minières, les affaissements miniers, de risques liés au travail se sont transformés en risques environnementaux. Les dégâts miniers étaient traités jusque-là en interne par les exploitants, et ils sont donc devenus une affaire des services de l'Etat lors de la remise des concessions minières. Ce transfert a été particulièrement vif dans le bassin ferrifère lorrain, aux couches peu profondes et donc plus propice que d'autres aux phénomènes d'affaissement. Les réactions locales à ces effondrements ont été d'autant plus virulentes qu'elles touchaient un territoire en crise. Les services de l'Etat ont avant tout cherché à apporter des réponses techniques et sectorielles pour prévenir les risques. Or les affaissements ont fait bouger bien plus que les maisons. Ils ont mis en mouvement des acteurs locaux issus d'une histoire politique, économique et sociale née des mines et de la sidérurgie et meurtries par la désindustrialisation. Au final, c'est précisément parce qu'il y avait un contexte territorial spécifique, avec la mobilisation de forces sociales encore chargées d'une histoire toute proche, qu'une dimension politique s'est imposée dans la gestion et le traitement de ces risques.

With the end of mining activity and the sale of miners' dwellings, the question of subsidence, hitherto an industrial hazard, became an environmental risk. The damage caused by mining, an issue previously addressed privately by the owners, became the responsibility of government services when mining concessions were handed over to the State. The handover was particularly delicate in the iron-ore basin of Lorraine, where the closeness of the ore to the surface made this area more liable to subsidence than others. Local reactions to the phenomenon were all the more heated as the territory was already in the throes of an economic crisis. The solutions sought by State services to safeguard against risk were primarily technical and sector-specific. However, it was not just houses that were 'moved' by the subsidence. It likewise provoked a reaction from local stakeholders who, with a political, economic and social heritage rooted in mining and the steel industry, had been bruised and battered by deindustrialisation. In the end, it was, in fact, due to a specific, territorial context, involving the mobilisation of social forces still deeply influenced by recent history, that the management and treatment of these risks took on a political dimension. 
INDEX

Mots-clés : affaissement minier, bassin ferrifère, risque minier, Plan de prévention des risques miniers (PPRM)

Keywords : Lorraine, mining subsidence iron-ore basin, mining risk, Mining Risk Prevention Plan (PPRM)

\section{AUTEUR}

\section{FRANÇOIS DUCHÊNE}

François Duchêne est architecte DPLG, urbaniste et géographe. Il est chargé de recherche du ministère de l'Écologie et du développement durable, et travaille au laboratoire RIVES, à l'École nationale des travaux publics de l'État, au sein de l'équipe « risques en milieu urbain ». Il a publié en 2002 Industrialisation et territoire. Rhône-Poulenc et la construction sociale de l'agglomération roussillonnaise chez l'Harmattan, en 2004, avec Christelle Morel Journel, De la culture du risque, aux éditions de l'Aube. Il prépare pour 2009 la publication de Cités ouvrières en devenir. Ethnographies d'anciennes enclaves industrielles aux PUSE. 